\title{
SALP Swarm Optimization Approach for Maximization The Lifetime of Wireless Sensor Network
}

\author{
Mohammed M. Ahmed
}

\begin{abstract}
In recent years, the maximization of a lifetime for wireless sensor networks is considered an important area for researchers. The wireless sensor networks (WSNs) contain two types of sensors that called sensor nodes and sink nodes which sensor node send information to the central node (sink node) that collected its data. Choosing the best location of sink node considered the critical problem that faces the lifetime of wireless sensor networks. In this paper, we propose a method that choosing best location of a sink node by applying Salp Swarm Algorithm (SSA) after determining sink node location we create transmission paths between the sink node and rest of nodes using Prim's minimum spanning tree to choose shortest paths. Accordingly, for fitness function that used to decrease energy consumption for a network. Simulation results clarify that our proposed algorithm that solves localization of sink node presents the best results for prolonging the network's lifetime compared to Cat Swarm Optimization algorithm (CSA) and Particle Swarm Optimization (PSO).
\end{abstract}

Keywords: Wireless sensor networks (WSNs), sink node placement, Salp swarm algorithm (SSA)..

\section{INTRODUCTION}

Nowadays, Wireless sensor networks (WSNs) are used in many of fields such as security surveillance, health, military, habitat monitoring, disaster management, and WSN have a large number of sensor nodes that monitor specific region and sink node which collect data that receive from sensor node in network [1]. The most important applications for WSNs is monitoring earthquake remotely before earthquake waves occurred in specific region [2] by choosing the best location of the central seismic station.

Many researchers are interested in single sink node localization to improve the network's lifetime. In [3] proposed the algorithm to optimize K-Nearest Neighbor (KNN) to choose best $\mathrm{k}$ number of neighbors for the sink node that is determined via whale optimization algorithm. In regards of the node localization, the determination of sink node location in a small WSNs based on cat swarm optimization algorithm (CSA) was proposed in [4] and compared with particle swarm

Manuscript received on 15 March 2021 | Revised Manuscript received on 22 March 2021 | Manuscript Accepted on 15 April 2021 | Manuscript published on 30 April 2021.

* Correspondence Author

Mohammed M. Ahmed*, Faculty of Computers and Artifcial Intelligence, University of Sadat City, Egypt. Email: mohammed.mostafa@fcai.usc.edu.eg

(C) The Authors. Published by Lattice Science Publication (LSP). This is an open access article under the CC-BY-NC-ND license (http://creativecommons.org/licenses/by-nc-nd/4.0/) optimization (PSO) in [5]. In [6] proposed binary version from single objective swarm is called whale optimization algorithm, that choose the best number of active nodes, inactive node and it's locations in the network but ensure all network is covering using breadth-first search to maximize the network's lifetime.

In [7] proposed the method by Yadav et al. that solve the clustering problem via PSO with using a distance of transmission for sensor nodes and residual energy. The best methodologies that saving energy for wireless sensor networks called topology control protocol (TC) such as the coverage and network's connectivity [8], [9] but Security is considered one of the problems that face TC [10].

Although the location of the sink node is one of the most important problems facing WSNs, many researches such as [10] presented a method for choose best of multiple sink nodes in LSWSNs. In most of the papers, the sensor node that deployed uniformly in a geometric region such ad rectangular area or circular area, the location of sink node will be located in the middle of the area such as in [11] that proposed P-Median Problem model. The Even though the sink node location is an obvious problem in WSNs, the literature has been dealt with sink node location rarely compared to other areas. Although the location of the sink node is one of the most important problems facing WSNs, many researchers such as [12] presented a method for choose best of multiple sink nodes in LSWSNs that choose best of multiple sink nodes locations. Also, in [13] proposed to allocate multiple sink nodes location in WSN and transmission path from all sensors to these multiple sink nodes.

This paper presents a new method that solves the localization of sink node in WSNs based on salp swarm algorithm (SSA) [14] called (SSA). This work proposes to solve the problem of choosing the best location single sink node Followed by a decrease in energy consumption and prolong the network's lifetime in WSNs. thereafter, uses the method of the greedy algorithm [15] such as Prim's algorithm [16] to create transmission paths from sink node to all rest of nodes in the network that regards a minimum spanning tree (MST) [17] algorithm which chooses shortest paths. Finally, the results of SSA is compared with other swarm optimization algorithms such as Cat Swarm Optimization algorithm (CSA) and Particle Swarm Optimization (PSO). In this paper introduces an effective method by combining the multi-objective technique with the bird swarm algorithm (BSA) to obtain a new method called MBSA[22].

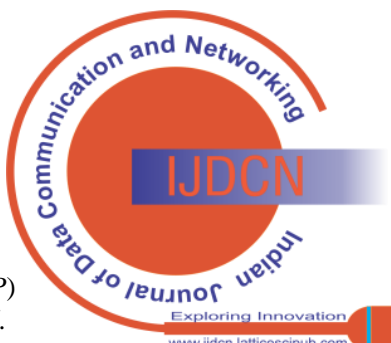


And we can then develop the proposed algorithm SSA-based sink node placement to become multi-objective algorithm to overcome many of the objectives that represent Pareto optimal

(PO) solutions [18] [19].

The structure of this paper is organized as follows. Section 2 presents an overview related to our methodologies. Section

3 introduces the proposed SSA-based sink node placement. The performance evaluation of the proposed algorithm with the existing algorithms is presented in Section 4. Conclusion are presented in Section 5.

\section{PRELIMINARIES}

\section{A. Network Model}

In this paper, WSNs have a set of sensor nodes and have one single sink node. All sensor nodes distributed randomly in an area called $\mathrm{R}$ that defined $\mathrm{R}=\mathrm{L}^{*} \mathrm{~L}$, where $\mathrm{L}$ represents the side length. this network is deployment by attaraya simulator that extracts a deployment network with coverage and connectivity. there are some of the assumptions for a network that follows: Firstly, assume there are set of sensor nodes that defined by $\mathrm{N}=\{\mathrm{n} 1, \ldots, \mathrm{nk}\}$. Secondly, all sensor nodes have different energy and the same communication range and same sensing range. Finally, there is a single sink node in the network that is located in a different location that chooses the best location for the sink node in this paper.

\section{B. Topology Control}

The Topology control (TC) [20] contains two types that called Topology Construction and Topology Maintenance. The first type Topology Construction is to construct miniature topology network of a smaller number of active nodes and minimizing transmission power. The second type Topology Maintenance aims to repair the constructed topology network with the collaboration of formerly inactive nodes. This maintenance procedure allows all nodes to participate within the network.

The target of TC protocols is maximizing the network's lifetime with the balance between energy consumption and radio interferences reductions. However, TC protocols have some drawbacks such as security for TC protocols Which affect negatively on the efficiency of the network such as protocols [10]. Most of the TC protocols that explained in researches such as A3 [8] and A3Cov [21] assume the sink node location is in the center of the network if the deployment of the network is uniformly so this case is not best result in normal life. So, in this paper proposes a contribution to extending the network's lifetime using salp swarm algorithm (SSA) based sink node placement to find the best location of sink node in the network.

\section{Salp Swarm Algorithm}

All Salp Swarm Algorithm (SSA) was proposed by Mirjalili et al. [14], and mathematically has been modeled as follows:

The leader position updated by the equation:

$X_{k}^{1}= \begin{cases}F_{k}+c_{1}\left(\left(u b_{k}-l b_{k}\right)\right) c_{2}-l b_{k} & \text { if } c_{3} \geq 0 \\ F_{k}+c_{1}\left(\left(u b_{k}-l b_{k}\right)\right) c_{2}-l b_{k} & \text { if } c_{3}<0\end{cases}$
Where $\mathrm{X}_{\mathrm{k}}{ }^{1}$ represents the position of the first leader salp during kth dimension, $F_{k}$ represents position source of food in the kth dimension, the upper bound of $k$ th dimension represented by $u b_{j}$, the lower bound of kth dimension represented by $\mathrm{lb}_{\mathrm{j}}$, and $\mathrm{c}_{1}, \mathrm{c}_{2}$, and $\mathrm{c}_{3}$ calculated by random numbers.

The coefficient $c_{1}$ is the most important parameter in SSA because it balances exploration and exploitation defined as follows:

$$
c_{1}=2 e^{-\left(\frac{4 l}{\pi}\right)^{2}}
$$

The current iteration represented by $\mathrm{l}$ and the maximum iteration represented by $\mathrm{L}$.

The position of the followers updated by an equation:

$x_{k}^{i}=\frac{1}{2} a t^{2}+v 0 t$

Where $i \geq 2, \mathrm{x}_{\mathrm{j}}^{\mathrm{i}}$ shows the position of $\mathrm{i}$ th follower salp in $\mathrm{k}$ th dimension, $\mathrm{t}$ is time, $\mathrm{v} 0$ is the initial speed, and $\mathrm{a}=\mathrm{v}_{\text {final }} /$ $\mathrm{v}_{0}$ where $\mathrm{v}=\mathrm{x}-\mathrm{x}_{0} / \mathrm{t}$.

$x_{k}^{i}=\frac{1}{2}\left(x_{k}^{i}+x_{k}^{i-1}\right)$

Where $i \geq 2$ and the position of $\mathrm{i}$ th follower salp in kth dimension represented by $x_{k}^{i}$. With Eq.1 and Eq.4.

\section{THE PROPOSED SINK NODE PLACEMENT USING SALP SWARM ALGORITHM}

This section describes the design of salp swarm algorithm for sink node placement (SSA-based sink node placement) as shown in Fig 1 and the detailed pseudo code of the SSA-based Sink node placement is illustrated in Algorithm 1.

Although most of the researches suppose that the location of the sink node in the center of the network such as A3, A3Cov topology control Protocols. this is not an optimal situation in real experiments. So better to improve the selection sink node location to save the network's coverage and operational lifetime

To select the location of the sink node in WSNs, the residual energy equipped on the sensor nodes are assumed to be randomly distributed network in this paper for finding the best location of the sink node. In our proposed SSA-based sink node placement, every salp represents a potential location of the sink node, and the salp with the best fitness value is capable to determine the final location of the sink node in the WSNs.

The steps for achieving the goal with SSA is listed as follows in Fig 1. This paper introduces a solution to the optimization problem, where the fitness function is minimizing optimal sink node located in a WSNs and reducing energy consumption.

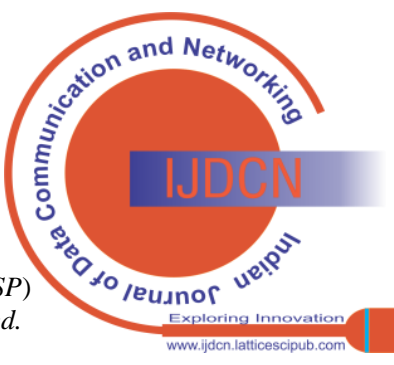




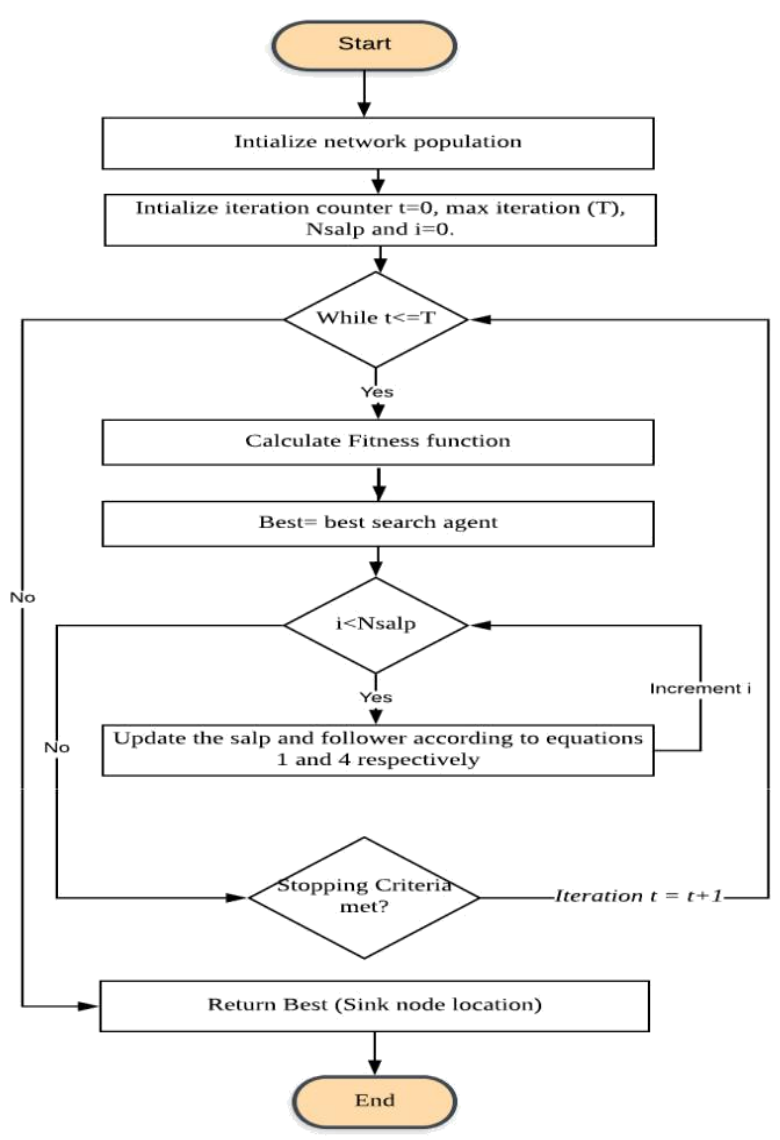

Figure 1: The architecture of the SSA-based sink node placement.

\section{Algorithm 1 The proposed SSA-based sink node} placement algorithm.

1: Input: A graph represents the nodes with energies

2: Output: Best location of sink node

3: Initialize the salp population $\mathrm{x}_{\mathrm{i}}$; $(\mathrm{i}=1,2, \ldots, \mathrm{n})$.

4: Initialize ub, lb.

5: Initializing start of iteration $\mathrm{t}=1$ and initialize Maximum of iteration $\mathrm{T}$.

6: while $\mathrm{t}<=\mathrm{T}$ do

7: Calculate the fitness function for each search agent (sink node) by Eq5.

8: $\quad$ Best= the best search agent.

9: Update $c_{1}$ b Eq.

10: for all search agents of $\mathrm{x}_{\mathrm{i}}$

11: if $\mathrm{i}==1$ then

12: Update sink node by Eq.1

13: $\quad$ Else $(|\mathrm{A}| \geq 1)$

14: Update follower of sink node by Eq.4

15: end if

16: EndFor

17: Amend the salps based on the upper and lower bounds of variables

18: end while

19: Return Best (Sink Node Location).

\section{FITNESS FUNCTION}

The performance of SSA evaluated via the location of the sink node and energy consumption in different algorithms such as CSA and PSO. Equation 5 is considered the fitness function of the proposed algorithm.

$f(x)=\alpha_{1} \frac{A N}{N}+\alpha_{2} \sum_{i=1}^{N_{\text {neighbor }}} E_{\text {neighbor }}+\alpha_{2}$ TDis
Where AN represents the number of active nodes, $\mathrm{N}_{\text {neighbo }}$ represents set of neighbors that served by sink node, and $E_{\text {neighbor }}$ represents energy for each sensor node that is considered neighbor for the sink node that selected and TDis is the total distances between the sink node and all sensor nodes and in Table 1 clarifies parameter setting.

\section{EXPERIMENTAL RESULTS AND DISCUSSION}

In this section discusses the experiment results for the proposed algorithm SSA compared to CSA and PSO. In Table 1 illustrates simulation parameters Which have been used in all experiments.

All the results are executed using the same PC that has settings which clarify in Table 2 .

Table 1. Atarraya simulation parameter.

\begin{tabular}{ll} 
Parameter & Value \\
& \\
\hline Deployment area & $600 \mathrm{~m} * 600 \mathrm{~m}$ \\
Number of nodes & $100,200, \ldots, 1000$ \\
Sensor node model & Simple \\
Node communication & range $100 \mathrm{~m}$ \\
Node sensing & range $20 \mathrm{~m}$ \\
Node location distribution & Uniform \\
Node energy distribution & Uniform \\
Max energy & 1000 milliamperes-hour(mA-h) \\
$\propto_{1}$ & 0.3 \\
$\propto_{2}$ & 0.1 \\
\hline
\end{tabular}

Table 2. The detailed settings.

\begin{tabular}{ll} 
Name & Detailed settings \\
\hline Software & \\
\hline $\begin{array}{l}\text { Operating system } \\
\text { Language }\end{array}$ & Windows 10 \\
Hardware & MATLAB R2015a \\
\hline Processor & Core(TM) i7-4500 \\
Frequency & $2.40 \mathrm{GHz}$ \\
Memory & $8 \mathrm{G}$ \\
Hard Disk & $500 \mathrm{~GB}$
\end{tabular}

The target from this experiment is to verify the proposed SSA algorithm and compare it with two algorithms CSA and PSO. In this experiment, in Table 3 depicts the running time of the proposed algorithm SSA is better than another algorithms CSA and PSO.

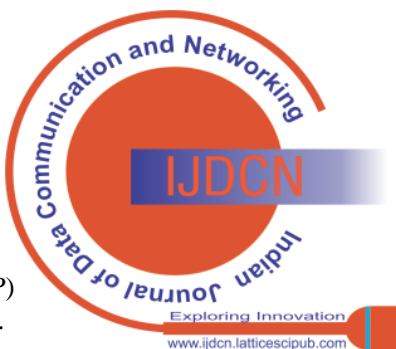


Table 3. Running Time for the different algorithms per second.

\begin{tabular}{llll}
\hline $\mathrm{N}$ & SSA (s) & CSA (s) & PSO (s) \\
\hline 100 & 196.9 & 396.9 & 395.3 \\
200 & 735.8 & 1135.8 & 1303.6 \\
300 & 1473.1 & 2073.1 & 2509.6 \\
400 & 3648.0 & 4448.0 & 5872.1 \\
500 & 5058.5 & 6058.5 & 8087.7 \\
600 & 6825.4 & 8025.4 & 10838.0 \\
700 & 9674.7 & 11074.7 & 15212.0 \\
800 & 11356.3 & 12956.3 & 17834.4 \\
900 & 14247.4 & 16047.4 & 22271.1 \\
10000 & 17450.0 & 19450.0 & 27174.9 \\
\hline
\end{tabular}

\section{A. Results and discussion}

Define All obtained results summarize energy consumption for compared algorithms SSA, CSA and PSO in Table 4. This table clarify proposed sink node placement using salp swarm algorithm overcome on other algorithms.

Table 4. Energy Consumption for the different algorithms.

\begin{tabular}{llll}
\hline $\mathrm{N}$ & SSA (s) & CSA (s) & PSO (s) \\
\hline 100 & 9102 & 10354 & 12855 \\
200 & 8932 & 11729 & 11847 \\
300 & 10296 & 13520 & 12843 \\
400 & 9347 & 11254 & 11935 \\
500 & 10352 & 13956 & 12739 \\
600 & 11732 & 14258 & 13654 \\
700 & 10368 & 13547 & 15326 \\
800 & 12635 & 13952 & 15466 \\
900 & 12864 & 14963 & 13756 \\
10000 & 11285 & 16302 & 15356 \\
\hline
\end{tabular}

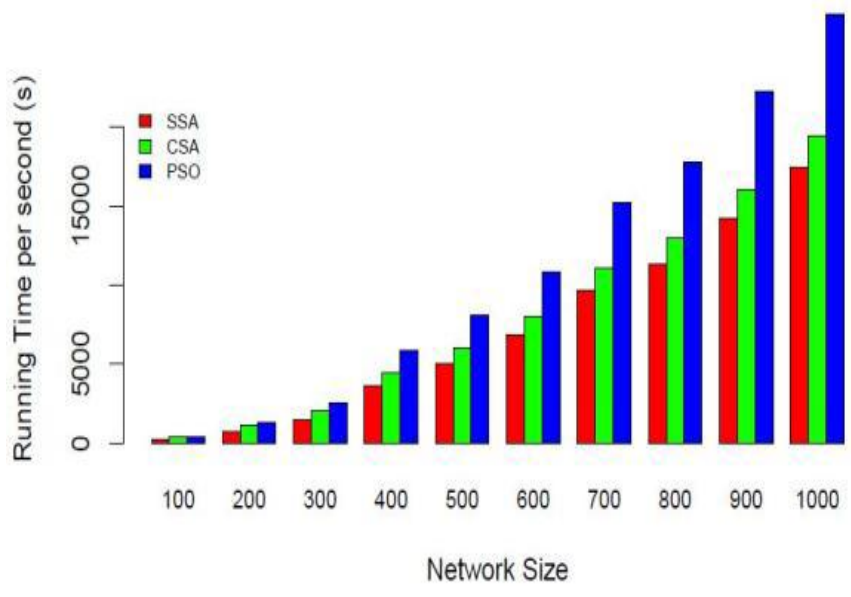

Fig. 2: Running Time for algorithms SSA, CSA and PSO for all network sizes.

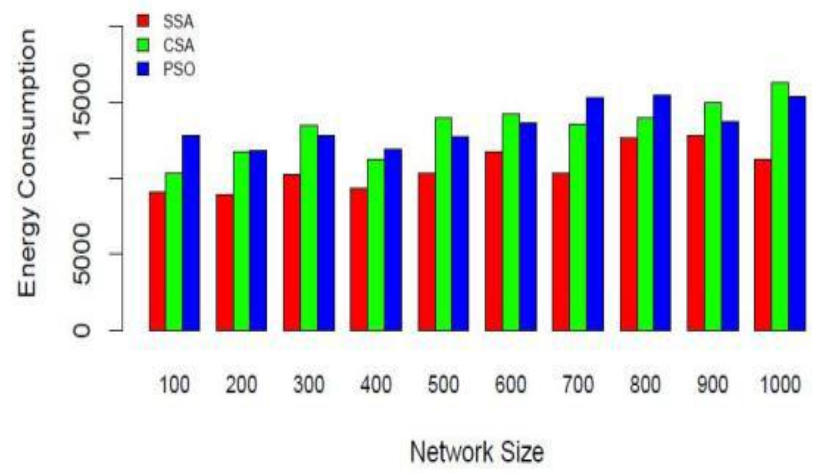

Fig. 3: Energy Consumption for algorithms SSA, CSA and PSO for all network sizes.

Fig 4 and 5 depicts position of sink node for 100 and 200 nodes respectively and clarify transmission path from each sink node that selected by each algorithm and the rest of sensor nodes in the network via minimum spanning tree [15] such as Prim's algorithm [16] that used with proposed SSA-based sink node placement that solves sink node placement. Note the results that appear with the proposed SSA when the number of nodes increased in the network give better results compared with the other algorithms CSA and PSO in terms of sink location and energy consumption.

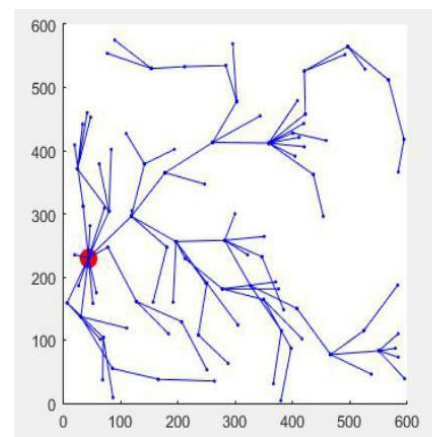

(a) SSA.

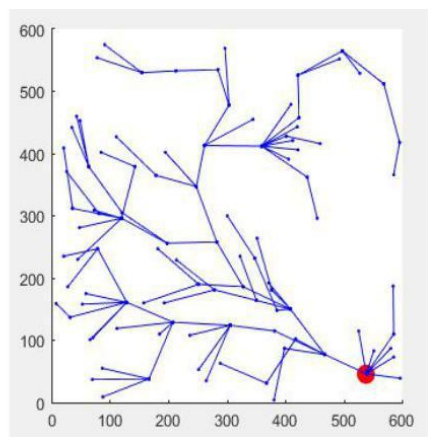

(b) CSA

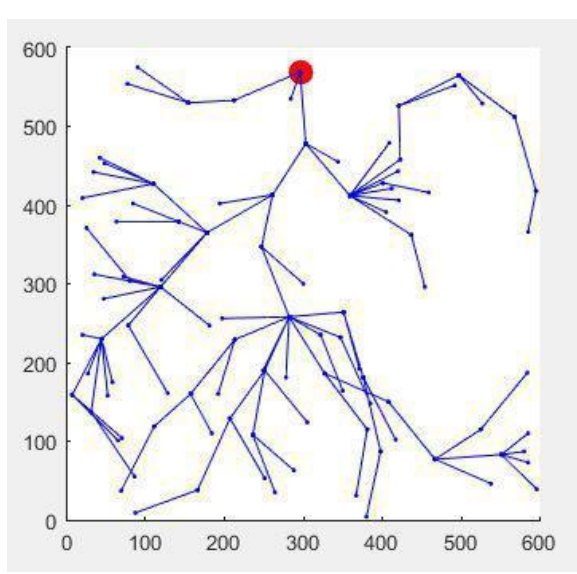

(c) PSO.

Fig. 4: Location of sink node in network size 100 for SSA, CSA and PSO.

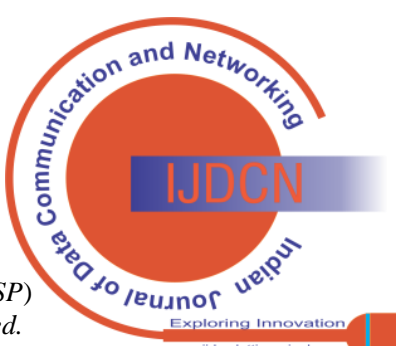




\section{CONCLUSION}

In this paper, the salp swarm algorithm (SSA) has been adapted to optimize the single sink node location wireless sensor networks (WSNs) with using Prim's minimum spanning tree to choose shortest paths that called to data transmission paths from the sink node to the rest of nodes in network Accordingly we propose a fitness function that use to reduce energy consumption for the network. According to the experiments that our proposed strategy executed for 10 different network size, the results prove that our method is better results for decreasing energy consumption compared with the other algorithms Cat Swarm Optimization algorithm (CSA) and Particle Swarm Optimization (PSO). To extend this contribution for future work for this direction of sink node placement. SSA can be adapted to improve using the multi-objective concept to overcome more than one objective for this direction of WSNs such as reducing energy and coverage and more of objectives.

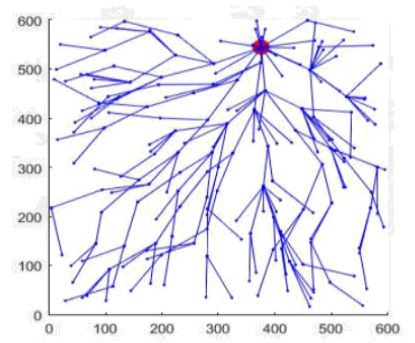

(a) SSA.

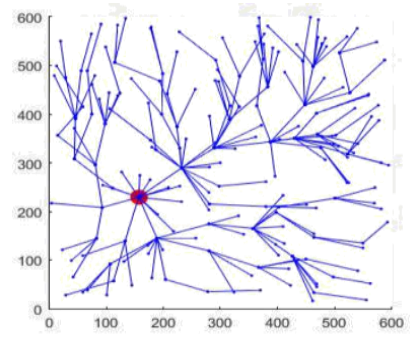

(b) CSA

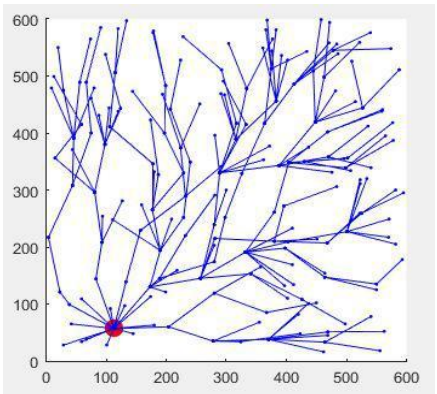

(c) PSO.

Fig. 5: Location of sink node in network size 200 for SSA, CSA and PSO.

\section{REFERENCES}

1. F. Akyildiz, W. Su, Y. Sankarasubramaniam, E. Cayirci, A survey on sensor networks, IEEE Communications magazine 40 (8) (2002) 102\{114. [CrossRef]

2. J. I. Espinosa-Ramos, et al., A new objective function to build seismic net-works using di erential evolution, in: Evolutionary Computation (CEC), 2012 IEEE Congress on, IEEE, 2012, pp. 1\{7. [CrossRef]

3. M. M. Ahmed, A. Taha, A. E. Hassanien, E. Hassanien, An optimized k-nearest neighbor algorithm for extending wireless sensor network lifetime, in: International Conference on Advanced Machine Learning Technologies and Applications, Springer, 2018, pp. 506\{515. [CrossRef]

4. V. Snasel, L. Kong, P. Tsai, J.-S. Pan, Sink node placement strategies based on cat swarm optimization algorithm, J. Netw. Intell 1 (2) (2016) $52\{60$.

5. M. M. Fouad, V. Snasel, A. E. Hassanien, Energy-aware sink node lo-calization algorithm for wireless sensor networks, International Journal of Distributed Sensor Networks 11 (7) (2015) 810356. [CrossRef]

6. M. M. Ahmed, E. H. Houssein, A. E. Hassanien, A. Taha, E. Hassanien, Maximizing lifetime of wireless sensor networks based on whale optimization algorithm, in: International Conference on Advanced
Intelligent Sys-tems and Informatics, Springer, 2017, pp. $724\{733$. [CrossRef]

7. R. Yadav, V. Kumar, R. Kumar, A discrete particle swarm optimization based clustering algorithm for wireless sensor networks, in: Emerging ICT for Bridging the Future-Proceedings of the 49th Annual Convention of the Computer Society of India CSI Volume 2, Springer, 2015, pp. $137\{144$. [CrossRef]

8. P. M. Wightman, M. A. Labrador, A3: A topology construction algorithm for wireless sensor networks, in: Global Telecommunications Conference, 2008. IEEE GLOBECOM 2008. IEEE, IEEE, 2008, pp. $1\{6$. [CrossRef]

9. P. Santi, Topology control in wireless ad hoc and sensor networks, ACM computing surveys (CSUR) 37 (2) (2005) 164\{194. [CrossRef]

10. M. M. M. Fouad, A. R. Dawood, M. M. Mostafa, Study of the e ects of pairwise key pre-distribution scheme on the performance of a topology control protocol.

11. A. Efrat, S. Har-Peled, J. S. Mitchell, Approximation algorithms for two optimal location problems in sensor networks, in: Broadband networks, 2005. BroadNets 2005. 2nd international conference on, IEEE, 2005, pp. $714\{723$.

12. E. I. Oyman, C. Ersoy, Multiple sink network design problem in large scale wireless sensor networks, in: Communications, 2004 IEEE International Conference on, Vol. 6, IEEE, 2004, pp. $3663\{3667$. [CrossRef]

13. H. Kim, Y. Seok, N. Choi, Y. Choi, T. Kwon, Optimal multi-sink position-ing and energy-e cient routing in wireless sensor networks, in: Interna-tional Conference on Information Networking, Springer, 2005, pp. $264\{274$. [CrossRef]

14. S. Mirjalili, A. H. Gandomi, S. Z. Mirjalili, S. Saremi, H. Faris, S. M. Mirjalili, Salp swarm algorithm: a bio-inspired optimizer for engineering design problems, Advances in Engineering Software 114 (2017) 163\{191. [CrossRef]

15. M. Hazewinkel, Greedy algorithm, encyclopedia of mathematics (2001).

16. B. Y. Wu, K.-M. Chao, Spanning trees and optimization problems (ex-cerpt).

17. D. Cheriton, R. E. Tarjan, Finding minimum spanning trees, SIAM Journal on Computing 5 (4) (1976) 724\{742. [CrossRef]

18. R. M. Rizk-Allah, A. E. Hassanien, A hybrid optimization algorithm for single and multi-objective optimization problems, in: Handbook of Re-search on Machine Learning Innovations and Trends, IGI Global, 2017, pp. 491\{521. [CrossRef]

19. A. Tharwat, E. H. Houssein, M. M. Ahmed, A. E. Hassanien, T. Gabel, Mogoa algorithm for constrained and unconstrained multi-objective opti-mization problems, Applied Intelligence (2017) $1\{16$. [CrossRef]

20. M. A. Labrador, P. M. Wightman, Topology Control in Wireless Sensor Networks: with a companion simulation tool for teaching and research, Springer Science \& Business Media, 2009.

21. P. M. Wightman, M. A. Labrador, A3cov: a new topology construction protocol for connected area coverage in wsn, in: Wireless Communications and Networking Conference (WCNC), 2011 IEEE, IEEE, 2011, pp. 522\{ 527. [CrossRef]

22. E. H. Houssein, M. M. Ahmed, M. A. Elaziz, A. A. Ewees and R. M. Ghoniem, "Solving Multi-Objective Problems Using Bird Swarm Algorithm," in IEEE Access, vol. 9, pp. 36382-36398, 2021, doi: 10.1109/ACCESS.2021.3063218. [CrossRef]

\section{AUTHORS PROFILE}

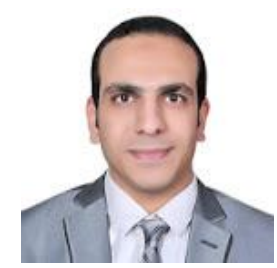

Mohammed M. Ahmed, was born in Minia, Egypt in 1989. He received his B.Sc. degree in 2010 from Faculty of Computers and Information, Information System Department, Minia University, Egypt. And he received M.Sc degree from FCI-Cairo University, Information System Department, Egypt, and assistant lecturer of Information systems at the Faculty of Computers and Artificial Intelligence, University of Sadat City. His research interests include Data mining, Wireless Sensor Networks, Swarm Optimization, Meta-heuristics, and Machine Learning.

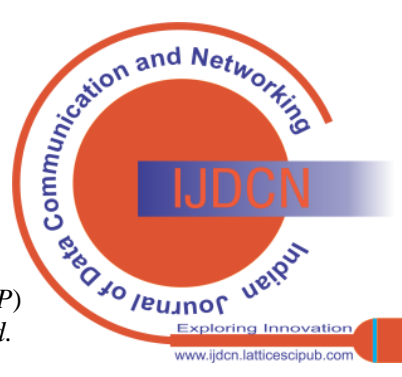

